\title{
Independence complexes and Edge covering complexes via Alexander duality
}

\author{
Kazuhiro Kawamura \\ Institute of Mathematics \\ University of Tsukuba, Ibaraki, Tsukuba 305-8571, Japan \\ kawamura@math.tsukuba.ac.jp
}

Submitted: Jun 8, 2010; Accepted: Feb 6, 2011; Published: Feb 14, 2011

Mathematics Subject Classiciation:05C10, 55P10, 05C05, 05C69, 05C99

\begin{abstract}
The combinatorial Alexander dual of the independence complex $\operatorname{Ind}(G)$ and that of the edge covering complex $\operatorname{EC}(G)$ are shown to have isomorphic homology groups for each non-null graph $G$. This yields isomorphisms of homology groups of $\operatorname{Ind}(G)$ and $\operatorname{EC}(G)$ with homology dimensions being appropriately shifted and restricted. The results exhibits the complementary nature of homology groups of $\operatorname{Ind}(G)$ and $\operatorname{EC}(G)$ which had been proved by Ehrenborg-Hetyei [10], Engström [11], and Marietti-Testa [16] for forests at homotopy level.
\end{abstract}

\section{Introduction and Preliminaries}

All graphs are assumed to be finite and simple. Topology of independence complexes has recently drawn much attention of various authors. See, for example, [2], [5] [6], [7], [9], [10], [11], [12], [14] [16], [15] etc. Ehrenborg and Hetyei [10] proved that the independence complex of a forest is either contractible or is homotopy equivalent to a sphere. Also Engström [11] and Marietti-Testa [15] independently gave algorithms to determine the dimension of the associated sphere (see [13] for another approach). Marietti and Testa [16] have shown that the homotopy types of the independence complex $\operatorname{Ind}(F)$ and the edge covering complex $\operatorname{EC}(F)$ of a forest $F$ are closely related: they are either both homotopy equivalent to spheres or both contractible. Furthermore, the dimensions of the associated spheres are both related to the domination number and differ by the number of components of $F$ [16, Theorem 4.16]. The referee of the first manuscript kindly pointed out that the method of Engström [11] can be applied to obtain these homotopy equivalences.

The result of the present paper shows that this complementary phenomenon is observed, to certain extent, for every non-null graph $G$ at homology level. It is pointed out in Proposition 2.4 that $\operatorname{susp}\left(\operatorname{Ind}(G)^{*}\right) \simeq \operatorname{susp}\left(\operatorname{EC}(G)^{*}\right)$, where $\operatorname{Ind}(G)^{*}$ and $\operatorname{EC}(G)^{*}$ 
denote the combinatorial Alexander duals of $\operatorname{Ind}(G)$ and $\operatorname{EC}(G)$ respectively. This and the Alexander duality provide us with isomorphisms of homology groups of $\operatorname{Ind}(G)$ and $\mathrm{EC}(G)$ in appropriately shifted and restricted dimensions (Theorem 2.5).

The result is a consequence of two theorems. One is due to Csorba [7]: the independence complex $\operatorname{Ind}\left(G_{2}\right)$ of the graph $G_{2}$, obtained from a graph $G$ by replacing each edge with a path of length 2 , is homotopy equivalent of the suspension $\operatorname{susp}\left(\operatorname{Ind}(G)^{*}\right)$ of the combinatorial Alexander dual of $\operatorname{Ind}(G)$. The other is due to Jonsson [12]: the independence complex of a bipartite graph is homotopy equivalent to the suspension of a simplicial complex defined in terms of adjacency relation of the graph (see below for the definition). The above theorem of Jonsson enables us to give another description of $\operatorname{Ind}\left(G_{2}\right)$ in terms of the independence complex of an associated bipartite graph with partite set $V(G)$ and $E(G)$, which yields the desired homotopy equivalence $\operatorname{Ind}\left(G_{2}\right) \simeq \operatorname{susp}\left(\operatorname{EC}(G)^{*}\right)$.

In the rest of this section, we make notational convention, give basic definitions and state auxiliary results. We follow [8] for terminology on graph theory. For a graph $G$, $V(G)$ and $E(G)$ denote the vertex set and the edge set of $G$ respectively. A graph with non-empty edge set is called a non-null graph. For a vertex $v$ of $G, N_{G}(v)$ denotes the set of all neighbors of $v$. For a subset $A$ of $V(G)$, the set $N_{G}(A)=\cup_{v \in A} N_{G}(v)$ is called the set of neighbors of $A$. A subset $I$ of $V(G)$ is said to be independent if, for each pair $u, v$ of distinct vertices of $I$, we have $u v \notin E(G)$. For a vertex $v$ and an edge $e$, the notation " $v \in e$ " means that $v$ is an end vertex of $e$. A subset $C$ of $E(G)$ is said to cover $G$ if, for each vertex $v \in V(G)$, there exists an edge $e$ of $C$ such that $v \in e$. Such subset $C$ is called an edge cover of $G$. A subset $D$ of $V(G)$ is a dominating set of $G$ if, for each vertex $v$ of $V(G) \backslash D$, there exists a vertex $u \in D$ such that $u v \in E(G)$.

For a graph $G, G_{2}$ is the graph obtained from $G$ by replacing each edge of $G$ by a path of length 2 [7]. Similarly, a graph $G_{n}$ is defined in [7] for $n \geq 2$, while we focus on $G_{2}$ here.

An abstract simplicial complex $K$ with a vertex set $V$ is a family of non-empty subsets of $V$ with the property: $\sigma \in K$ and $\tau \subset \sigma$ imply $\tau \in K$. We identify $K$ with its geometric realization, which causes no confusion. For two simplicial complexes $K$ and $L, K \cong L$ means that they are isomorphic as simplicial complexes. The suspension over a simplicial complex $K$ is denoted by $\operatorname{susp}(K)$. For a simplicial complex $K$ with vertex set $V$, the combinatorial Alexander dual $K^{*}$ is the simplicial complex defined by

$$
K^{*}=\{\sigma \mid \sigma \subset V, V \backslash \sigma \notin K\} .
$$

When $K$ is not the simplex with the vertex set $V, K$ is regarded as a subset of $S^{|V|-2}$ dimensional sphere and it is known that $K^{*}$ is homotopy equivalent to $S^{|V|-2} \backslash K([7])$.

For two simpicial complexes $K$ and $L, K \simeq L$ means that $K$ and $L$ have the same homotopy type. For a simplicial complex $K, \tilde{\mathrm{H}}_{i}(K)$ and $\tilde{\mathrm{H}}^{i}(K)$ denote the reduced singular homology and singular cohomology groups of $K$ with integer coefficients respectively. We make a convention that $\tilde{\mathrm{H}}_{i}(K)=\tilde{\mathrm{H}}^{i}(K)=0$ for each $i<0$. It is well-known [17]

$$
\tilde{\mathrm{H}}_{i}(\operatorname{susp}(K)) \cong \tilde{\mathrm{H}}_{i-1}(K) \text { and } \tilde{\mathrm{H}}^{i}(\operatorname{susp}(K)) \cong \tilde{\mathrm{H}}^{i-1}(X)
$$

for each $i \geq 0$. 
For a graph $G$, the following two simplicial complexes are the subject of our study. The independence complex $\operatorname{Ind}(G)$ with the vertex set $V(G)$ is defined by

$$
\operatorname{Ind}(G)=\{\sigma \mid \emptyset \neq \sigma \subset V(G), \quad \sigma \text { is independent }\} .
$$

The edge covering complex $\operatorname{EC}(G)$ with the vertex set $E(G)$ is defined by

$$
\mathrm{EC}(G)=\{F \mid \emptyset \neq F \subset E(G), \quad E(G) \backslash F \text { is an edge cover of } G\} .
$$

We apply the Alexander duality in the following form to derive the desired homology equivalence:

Theorem 1.1 ([17], Theorem 71.1) For each proper subcomplex $K$ of the $n$-dimensional sphere $S^{n}$, we have an isomorphism

$$
\tilde{\mathrm{H}}_{n-1-i}\left(S^{n} \backslash K\right) \cong \tilde{\mathrm{H}}^{i}(K)
$$

for each $i=-1, \ldots, n$. In particular, for each non-simplex simplicial complex $K$ with $n$ vertices, being regarded as a subcomplex of $S^{n-2}$, we have

$$
\tilde{\mathrm{H}}_{n-3-i}\left(K^{*}\right) \cong \tilde{\mathrm{H}}^{i}(K)
$$

for each $i=-1, \ldots, n-2$, where $K^{*}$ denotes the combinatorial Alexander dual of $K$.

Now we recall a theorem due to Jonsson [12]. For a bipartite graph $B=B(X, Y)$ with partite sets $X$ and $Y$, we define simplicial complexes $\Gamma_{X}$ and $\Gamma_{Y}$ as follows:

$$
\begin{aligned}
& \Gamma_{X}=\left\{\sigma \subset X \mid Y \backslash N_{B}(\sigma) \neq \emptyset\right\} \quad \text { and } \\
& \Gamma_{Y}=\left\{\tau \subset Y \mid X \backslash N_{B}(\tau) \neq \emptyset\right\} .
\end{aligned}
$$

Theorem 1.2 ([12], Theorem 3.2) For each bipartite graph $B$ with partite sets $X$ and $Y$, we have the following homotopy equivalences.

$$
\begin{aligned}
\operatorname{Ind}(B) & \simeq \operatorname{susp}\left(\Gamma_{X}\right) \\
& \simeq \operatorname{susp}\left(\Gamma_{Y}\right)
\end{aligned}
$$

\section{Result}

For a graph $G$, we define a bipartite graph $B_{G}=B(V(G), E(G))$ with the partite sets $V(G)$ and $E(G)$ by the following: for $v \in V(G)$ and $e \in E(G)$,

$$
v e \in E\left(B_{G}\right) \text { if and only if } v \text { is an end vertex of } e \text {. }
$$

It is easy to see that the graph $B_{G}$ is isomorphic to $G_{2}$. For the graph $B_{G}$ defined above, the simplicial complex $\Gamma_{E(G)}$ in Theorem 1.2 is written as follows:

$$
\begin{aligned}
\Gamma_{E(G)} & =\left\{F \subset E(G) \mid V(G) \backslash \cup_{e \in F} N_{B_{G}}(e) \neq \emptyset\right\} \\
& =\{F \subset E(G) \mid E(G) \backslash F \text { is not an edge cover of } G\} .
\end{aligned}
$$


The definition of the combinatorial Alexander dual immediately implies

$$
\Gamma_{E(G)}=E C(G)^{*}
$$

and hence by Theorem 1.2 we have

Lemma 2.1 For each non-null graph $G$, we have a homotopy equivalence $\operatorname{Ind}\left(G_{2}\right) \cong$ $\operatorname{Ind}\left(B_{G}\right) \simeq \operatorname{susp}\left(E C(G)^{*}\right)$.

Remark 2.2 The dominance complex $\operatorname{Dom}(G)$ of a graph $G$ is a simplicial complex with the vertex set $V(G)$ defined as follows.

$$
\operatorname{Dom}(G)=\{\sigma \mid \emptyset \neq \sigma \subset V(G) \text { and } V(G) \backslash \sigma \text { is a dominating set of } G\} .
$$

As in the above argument, we have the following inclusion

$$
\operatorname{Dom}(G)^{*} \subset \Gamma_{V(G)}
$$

Now we recall the following theorem due to Csorba [7]:

Theorem 2.3 ([7], Theorem 6) For each graph $G$, we have the following homotopy equivalence:

$$
\operatorname{Ind}\left(G_{2}\right) \simeq \operatorname{susp}\left(\operatorname{Ind}(G)^{*}\right) .
$$

Combining Lemma 2.1 with Theorem 2.3, we have the following:

Proposition 2.4 For each non-null graph $G$, we have the following homotopy equivalence:

$$
\operatorname{susp}\left(\operatorname{Ind}(G)^{*}\right) \simeq \operatorname{susp}\left(\operatorname{EC}(G)^{*}\right) .
$$

The above result is applied to prove homology isomorphisms mentioned in the introduction. Following [16], let $\kappa(G)=|V(G)|-|E(G)|$. When $G$ is a forest, $\kappa(G)$ is the number of components of $G$.

Theorem 2.5 For each non-null graph $G$ and for each $i$ with $\max (-1, \kappa(G)-1) \leq i \leq|V(G)|-2$, we have an isomorphism:

$$
\tilde{\mathrm{H}}_{i}(\operatorname{Ind}(G)) \cong \tilde{\mathrm{H}}_{i-\kappa(G)}(\operatorname{EC}(G)) .
$$

Proof. Let $n=|V(G)|$ and $m=|E(G)|$ so that $\kappa(G)=n-m$. The simplices with the vertex set $V(G)$ and with the vertex set $E(G)$ are denoted by $\Delta_{V(G)}$ and $\Delta_{E(G)}$ respectively. Notice that $\operatorname{dim} \Delta_{V(G)}=n-1$ and $\operatorname{dim} \Delta_{E(G)}=m-1$. In particular, the boundary complexes $\partial \Delta_{V(G)}$ and $\partial \Delta_{E(G)}$ are homeomorphic to $(n-2)$ - and $(m-2)$ dimensional spheres. Since $G$ is a non-null graph, we see that $\operatorname{Ind}(G) \subset \partial \Delta_{V(G)}$. Also it is easy to see that $\operatorname{EC}(G) \subset \partial \Delta_{E(G)}$.

By Theorem 1.1, we have the following isomorphisms of homology groups

$$
\tilde{\mathrm{H}}_{i}(\operatorname{Ind}(G)) \cong \tilde{\mathrm{H}}^{n-3-i}\left(\operatorname{Ind}(G)^{*}\right)
$$


for each $i$ with $-1 \leq i \leq n-2$, and

$$
\tilde{\mathrm{H}}_{i}(\mathrm{EC}(G)) \cong \tilde{\mathrm{H}}^{m-3-i}\left(\mathrm{EC}(G)^{*}\right)
$$

for each $i$ with $-1 \leq i \leq m-2$. For each $i$ with $\max (-1, n-m-1) \leq i \leq n-2$, the desired isomorphism is obtained by a sequence of isomorphisms as follows:

$$
\begin{aligned}
\tilde{\mathrm{H}}_{i}(\operatorname{Ind}(G)) & \cong \tilde{\mathrm{H}}^{n-3-i}\left(\operatorname{Ind}(G)^{*}\right) \quad(\text { by }(2)) \\
& \cong \tilde{\mathrm{H}}^{n-2-i}\left(\operatorname{susp}\left(\operatorname{Ind}(G)^{*}\right)\right) \quad(\text { by }(1)) \\
& \cong \tilde{\mathrm{H}}^{n-2-i}\left(\operatorname{susp}\left(\operatorname{EC}(G)^{*}\right)\right) \quad(\text { by Proposition } 2.4) \\
& \cong \tilde{\mathrm{H}}^{n-3-i}\left(\operatorname{EC}(G)^{*}\right) \quad(\text { by }(1)) \\
& \cong \tilde{\mathrm{H}}_{(m-2-1)-(n-3-i)}(\operatorname{EC}(G)) \quad(\text { by }(3)) \\
& =\tilde{\mathrm{H}}_{m-n+i}(\operatorname{EC}(G))=\tilde{\mathrm{H}}_{i-\kappa(G)}(\operatorname{EC}(G)) .
\end{aligned}
$$

This completes the proof.

As is mentioned in Section 1, Results of Ehrenborg-Hetyei ([10]), Engström ([11]) and Marietti - Testa ([15] and [16]) tell us that for each forest $F, \operatorname{Ind}(F)$ and $\operatorname{EC}(F)$ are either both contractible, or both homotopy equivalent to spheres. Moreover, if $\operatorname{Ind}(F)$ is

not contractible, then we have homotopy equivalences: $\operatorname{Ind}(F) \simeq S^{\gamma(F)-1}$ and $\operatorname{EC}(F) \simeq$ $S^{\gamma(F)-1-\kappa(F)}$, where $\gamma(F)$ is the domination number of $F$ :

$$
\gamma(F)=\min \{|D| \mid D \text { is a dominating set of } F\} .
$$

Since $\gamma(F) \geq \kappa(F)$, we may apply Theorem 2.5 to confirm the isomorphism $\tilde{\mathrm{H}}_{\gamma(F)-1}(\operatorname{Ind}(F)) \cong \tilde{\mathrm{H}}_{\gamma(F)-1-\kappa(F)}(\mathrm{EC}(F))(\cong \mathbb{Z})$. Theorem 2.5 exhibits that this complementary phenomenon is observed for general (non-null) graph at homology (hence a weaker) level with homology dimensions being shifted and restricted.

Acknowledgment. The author expresses his deep gratitude to the referee who pointed out errors in the first manuscript and also made very helpful comments in the revision of the paper.

\section{References}

[1] E. Babson and D.N. Kozlov, Proof of the Lovász conjecture, Ann. Math. 165 (2007), 965-1007.

[2] J.A. Barmak, Star clusters in independence complexes of graphs, preprint.

[3] A. Björner, Topological Methods, in Handbook of Combinatorics, Vol.2, R.Graham M.Grötschel - L. Lovász (eds), North-Holland (1995), 1819-1872.

[4] A. Björner, L.M. Butler and A.O. Matveev, Note on a combinatorial application of Alexander duality, J. Combinatorial theory, Ser.A 80 (1997),163-165. 
[5] M. Bousqet-Mélou,S. Linusson and E. Nevo, On the independence complex of squaregrid, J. Algebraic Comb. 27 (2008), 423-450.

[6] B. Braun, Independence complexes of Stable Kneser graph, arXiv:0912.0720

[7] P. Csorba, Subdivision yields Alexander duality on independence complexes, Electronic J. Comb. 16 (2) (2009) $\sharp R 11$.

[8] R. Diestel, Graph Theory, third edition, GTM 173, Springer, 2005.

[9] A. Dochtermann and A. Engström, Algebraic properties of edge ideals via combinatorial topology, Electron. J. Comb. 16 (2009), No.2, Research Paper 2.

[10] R. Ehrenborg and G.Hetyei, The topology of the independence complexes, European J. Comb. 27 (2006), 906-023.

[11] A. Engström, Independence complexes of claw-free graphs, European J. Comb. 29 (2008), 234-241.

[12] J. Jonsson, On the topology of independence complexes of triangle-free graphs, preprint.

[13] K. Kawamura, Homotopy types of independence complexes of forests, Cont. Discrete Math. 5 (2010), 67-75.

[14] D. Kozlov, Combinatorial Algebraic Topology, Algorithm and Computations in Math. 21 (2008), Springer.

[15] M. Marietti and D. Testa, Cores of simplicial complexes, Discrete and Computational Geometry 40 (2008), 444-468.

[16] M. Marietti and D. Testa, A uniform approach to complexes arising from forests, Elect. J. Comb. 15 (2008), $\sharp R 101$.

[17] J. R. Munkres, Elements of Algebraic Topology, Addison-Wesley, 1984. 